\title{
Implementation of a Hepatitis C Collaborative Drug Therapy Management Service in an HIV Ambulatory Care Clinic
}

Maria A. Sorbera, PharmD, AAHIVP, BCACP ${ }^{1,2, a}$; Cathleen Mathew, PharmD, AAHIVP ${ }^{1, a, b}$; Shannon Christy Ramdeen PharmD, BCACP

${ }^{1}$ Department of Pharmacy, The Brookdale Hospital and Medical Center, Brooklyn, NY 11212

${ }^{2}$ Long Island University Arnold \& Marie Schwartz College of Pharmacy and Health Sciences, Brooklyn, NY 11212

${ }^{a}$ C.M. and M.A.S. contributed equally to this work

${ }^{b}$ Present affiliation: Fidelis Care, Rego Park, Queens 11374

\begin{abstract}
Purpose: Being the leading cause of liver disease and hepatocellular carcinoma, the hepatitis C virus (HCV) has become a growing public health threat within the United States. Of those individuals infected with human immunodeficiency virus (HIV), approximately $25 \%$ are co- infected with HCV. Establishment of a hepatitis C collaborative drug therapy management (CDTM) service can expand the role of pharmacists in an interdisciplinary team improving health outcomes for an underserved HIV/HCV co-infected patient population.
\end{abstract}

Summary: In 2016, physicians and pharmacists identified a need to establish Hepatitis C pharmacy services in an HIV clinic. Through the recent establishment of CDTM and implementation of pharmacy services, pharmacists are now given the autonomy to manage patients co-infected with HIV/HCV. It is believed that the addition of a clinical pharmacist to an interdisciplinary team will lead to improved SVR rates, adherence, and clinic revenue, as well as decreased ADRs and drug-drug interactions among co-infected patients.

Conclusion: Implementation of HCV pharmacy services directed towards HIV/HCV co- infection is an essential patient-centered strategy addressing complex medication-related problems in an attempt to achieve optimal therapeutic outcomes. A single-centered, prospective study to evaluate HCV pharmacy services at the Treatment for Life Center clinic is currently ongoing.

Keywords: Hepatitis C, Clinical Pharmacy Service, Public Health, Collaborative Drug Therapy Management

\section{Key Points}

1. Hepatitis $\mathrm{C}$ is a growing public health crisis that requires an interdisciplinary patient-centered approach to care.

2. Clinical pharmacists are uniquely positioned to assist in the management of HCV patients, specifically in promoting preventative measures, providing education to improve medication adherence, assisting in initiating $\mathrm{HCV}$ therapy, managing drug-drug interactions and adverse events, monitoring treatment responses, and facilitating medication acquisition.

3. The true role of pharmacists in assisting in the management of chronic disease states, such as HCV, is brought to its true potential through the establishment of collaborative drug therapy management.

Corresponding author: Maria A. Sorbera, PharmD, AAHIVP, BCACP, Assistant Professor of Pharmacy Practice

Arnold \& Marie Schwartz College of Pharmacy and Health Sciences Long Island University

Brooklyn, New York 11201

Ambulatory Care Faculty Preceptor, The Brookdale Hospital

Medical Center, Brooklyn, NY 11212

E-mail: maria.sorbera@liu.edu

\section{Background}

Often referred to as the "silent epidemic," the hepatitis C virus (HCV) has emerged as a major public health crisis in the United States. It is suspected that there are, at present, up to 9 million people in the country both acutely and chronically infected with HCV. Approximately 75 to $85 \%$ of acutely infected patients advance to chronic infection leading to possible complications of cirrhosis and/or hepatocellular carcinoma. ${ }^{1} \mathrm{HCV}$-related mortality is expected to rise and peak somewhere between 2030 and 2035, with an estimated 36,100 deaths. ${ }^{2}$ With treatment factored in, this mortality rate is likely to be lower. These statistics emphasize the need for increased HCV screening efforts among healthcare professionals and institutions as well as improved transitions into care. Considering that about one quarter of HIV-infected individuals are also infected with HCV, it is essential for HIV providers to be knowledgeable in the management of both disease states. ${ }^{3}$ Co-infection with HIV accelerates liver disease progression warranting the need for prompt screening and treatment of $\mathrm{HCV}^{4}$

The treatment of chronic HCV has undergone an evolution in the last few years with the development of direct-acting antivirals (DAAs). Compared to former interferon (IFN) and ribavirin (RBV) based regimens, DAAs are oral treatment options that when used in recommended combinations are associated with improved efficacy and tolerance with 
decreased durations of therapy. Results of clinical trials suggest that these regimens routinely achieve a cure, defined as a sustained virologic response 12 weeks post treatment (SVR12), in $\geq 90 \%$ of individuals including those who are treatment experienced and/or cirrhotic. ${ }^{5}$ The development of this novel class of medications finally allows healthcare providers to effectively combat the silent epidemic of HCV. However, this fight does not come without a steep cost.

In comparison to former HCV therapies, DAAs are associated with greater wholesale acquisition costs ranging from $\$ 650$ to $\$ 1,125$ per day of therapy. ${ }^{6,7}$ The actual price paid by the patient may be significantly lower due to third party contracts, manufacturer rebates, and discounts. Additionally, the process of acquiring third-party prior authorization approvals for DAAs can potentially delay treatment. Healthcare professionals involved with the treatment of HCV must stay up-to-date with the different requirements and restrictions of various insurance plans. Along with medication approvals, ongoing education within clinics and institutions is essential in order for providers to stay abreast the present and rapidly evolving treatment regimens.

With the newly approved DAAs, healthcare is not at a paucity of HCV treatment options. As DAAs are all efficacious with similar cure rates when used in recommended combinations, the determining factors when selecting a regimen are patientspecific. Providers and pharmacists must take into account differences in drug pharmacokinetics and dynamics, as well as potential clinically significant drug-drug interactions (CSDDIs). In an interdisciplinary setting, pharmacists are able to assist in initiating HCV therapy, manage CSDDIs and adverse events, monitor treatment responses, facilitate medication acquisition, promote preventative measures to reduce HCV transmission and re-infection, and provide education to improve medication adherence. This role is brought to its true potential through the establishment of collaborative drug therapy management (CDTM) increasing the autonomy of the pharmacist.

The involvement of pharmacists in anticoagulation services, HIV care, cystic fibrosis, and diabetes has been shown to increase adherence, reduce pill burden and dosing frequency, and decrease medication-related errors. ${ }^{8}$ Johnson and colleagues found that pharmacists can reduce drug-related morbidity and mortality for $28.2 \%$ of hospital admissions with an estimated savings of $\$ 76.6$ billion in an ambulatory setting. ${ }^{9}$ In a pilot study retrospectively evaluating outcomes from 2002-2004 in a pharmacist-managed HCV clinic at the Los Angeles Department of Veterans Affairs (VA) Healthcare System, it was concluded 17 out of 27 enrolled patients (63\%) achieved SVR. ${ }^{10}$ During the time of this pilot study, DAAs were not yet approved. Therefore, the lower SVR rates achieved were similar to the real-world cure rates of older regimens. In 2017, Yang and colleagues assessed the economic and clinical outcomes of pharmacy-led HCV utilization management in a VA Medical Center. In this retrospective study, a total of 372 patients were included in which the majority received ledipasvir/sofosbuvir or ombitasvir/paritaprevir/ritonavir with dasabuvir \pm ribavirin. Overall, the SVR rate achieved was $94.1 \%$. Investigators concluded the findings were suggestive that pharmacist-led HCV DAA utilization management is an important factor in costs and cure rates. ${ }^{11}$ Although research in this field is limited, the role clinical pharmacists can play to assist in improving patient-directed care includes education and counseling, managing drug-drug interactions, monitoring for efficacy and toxicities, and assisting in providing affordable medications to patients.

The initiation of CDTM services through a collaborative practice agreement (CPA) between pharmacists and health care providers in an HIV/HCV infected population can offer great benefits in both hospital and patient-centered health outcomes. Legalized in New York State in 2011, CDTM is currently limited to teaching and general hospitals, including any diagnostic center, treatment center or hospital-based outpatient department. ${ }^{12}$

Brookdale Hospital and Medical Center (BHMC) is a 530-bed tertiary care treatment facility with more than 50 specialty clinics including the Treatment for Life Center (TLC). Designated as a New York State (NYS) AIDS Center in 1993, the clinic provides a wide range of services to approximately 750 patients including HIV testing and counseling, treatment and partner notification, case management, as well as pharmacy, laboratory and social work services. Located in East New York, Brooklyn, the TLC cares for a highly underserved patient population. The incidence of HIV in Brooklyn is $6 \%$ lower than the incidence for New York City as a whole; however, wide disparities exist within the borough. East New York has been identified as a neighborhood that has one of the highest incidences of HIV and HIV/AIDS with lower rates of viral suppression. ${ }^{13,14}$

To aid in increasing viral suppression rates and improve treatment outcomes for other chronic disease states, the TLC utilizes an interdisciplinary approach to patient care. With the emergence of DAAs, the clinic developed a need for HCV services. As a direct response to this need, pharmacy established an HCV clinic with the goal to achieve high SVR rates and decrease HCV- associated morbidity and mortality within this medically underserved community. This article will describe the implementation process of hepatitis $C$ pharmacy services through a CPA at the TLC.

\section{Implementation of Hepatitis C Pharmacy Services Prior to the establishment of a CPA, the primary model for pharmacy services at the TLC was a walk-in medication adherence clinic in which scheduled appointments were not}


often made. Providers referred patients to the clinic who were identified as non-adherent, and these patients were often enrolled in pill tray services. On average, 120-180 patients were enrolled in these services. As HIV, along with several other chronic disease states were managed in the TLC, patients would be on multiple medications placing them at an increased risk for non-adherence and medication-related adverse events. On a regular basis, the clinic ambulatory care pharmacist conducted extensive chart reviews in order to identify medication interventions. However, without a formal agreement, interventions were dependent on follow-up time with the physicians due to the pharmacists' lack of autonomy. Allowing clinical pharmacists to provide a comprehensive medication management service optimized through a CPA was proposed, particularly in managing those co-infected with HCV.

To prepare for the development of a CPA, the pharmacists in the TLC attended professional development activities aimed at establishing and sustaining ambulatory care clinics, and consulted various healthcare professionals who practice under CDTM to obtain their professional guidance. Informal and formal verbal discussions occurred between the clinical pharmacists and BHMC physicians to ensure internal needs were mutually understood between both parties. The needs of the clinic expressed by providers included medication counseling and management specifically for the co-infected population. Discussions also took place with administrators, nurse practitioners, social workers and patient navigators, and the agreement included all noted suggestions. For 3 months, the pharmacy department held several meetings to review and edit drafts.

In January 2016, a final CPA draft was completed and approved by pharmacy. Following this, a meeting was held with all clinic providers to present the agreement and privileges to be granted. The importance of the agreement was unanimous, and proceeding minor edits, was accepted by the TLC physicians, administration, the institution's Legal Department, the Medical Executive Committee, and the Chief Medical Officer. Through the approval by all parties, the TLC CPA was the first formal practice agreement at the BHMC implementing CDTM and providing significantly greater autonomy to pharmacists.

The approved CPA established a documented policy for medication management with a scope exceeding beyond HCV management. Other disease states in the protocol include HIV and related co-infections, asthma, COPD, diabetes, heart failure, hypertension, hyperlipidemia, and nicotine dependence. Prescribers may refer patients for other disease states and services outside of those specifically listed, such as therapeutic drug monitoring. For all referred patients under the CPA, pharmacists may initiate, modify, discontinue and refill medications, order and evaluate laboratory tests, obtain vital signs, and collect and review patient histories. Within 24 hours of a patient care encounter, the pharmacist is required to communicate all treatment plans via the electronic medical record (EMR), which is then co-signed by the overseeing physician. The managing clinical pharmacists of these encounters are board-certified in ambulatory care and received their CDTM certification. Future pharmacists practicing under CDTM in the TLC will be required to have or have plans to obtain their board and CDTM certifications.

\section{Clinic Operation}

Patients are referred to HCV pharmacy services by their TLC physician if they are 18 years or older and are anticipated to start HCV treatment. Enrolled patients are then assigned an ambulatory care clinical pharmacist who will consistently follow the patient to ensure continuity of care. To standardize the care that is delivered to each patient, the clinic pharmacists utilize the Pharmacists' Patient Care Process (Fig. 1).

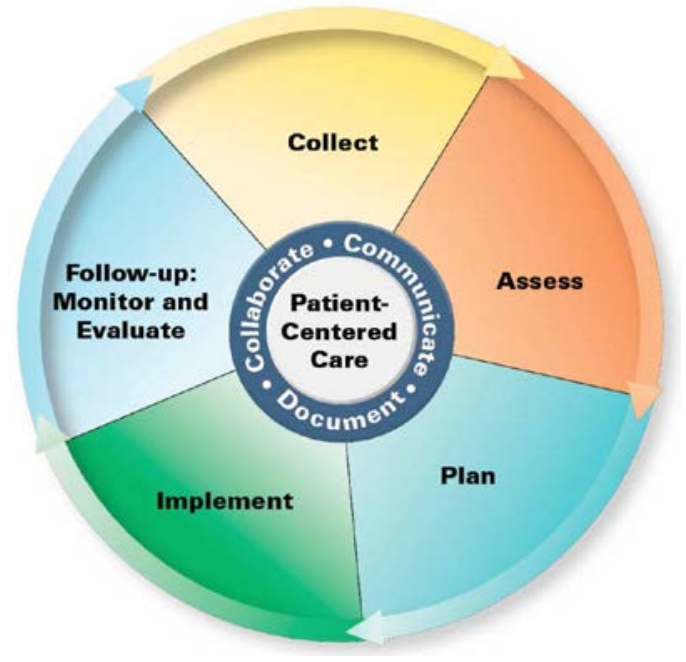

Figure 1: Pharmacists' Patient Care Process: A standardized process that aims to promote consistency across the profession and assist in achieving optimal health and medication-related outcomes. Released by the Joint Commission of Pharmacy Practitioners (JCPP). The Pharmacists' Patient Care Process JCPP. https://jcpp.net/patient-care-process/. Accessed September 17, 2017.

Prior to the initial visit, the pharmacist accesses BHMC's EMR, $\mathrm{EPIC}^{\mathrm{TM}}$, to review the patient's medical history, co-morbid conditions, laboratory tests, and to perform medication reconciliation. During the extensive review, pertinent information to HCV is gathered, including genotype, HCV RNA viral loads, stage of fibrosis and/or cirrhosis, and treatment history. Any further laboratory data that is warranted will be ordered and assessed by the pharmacist. Once all necessary information is obtained, the possible treatment options for the patient are determined in preparation for the initial visit. These options are communicated with the enrolled patient's 
TLC physician and a collaborative decision is made for the most appropriate regimen.

At the initial patient encounter, written consent is obtained from the patient stating he/she would like to receive HCV treatment through pharmacy services. Education is provided on the disease state, treatment options, and most importantly, on adherence. It is critical that the patient's readiness for treatment and ability to adhere to medication regimens is assessed prior to initiating therapy. The purpose of this assessment is not to deny treatment, but rather identify barriers and optimize support. Within the TLC, potential barriers that may exist include the complexity of the medication regimens, polypharmacy, patient-provider relationships, and patient-specific factors. The NYC Hep C Task Force developed an online tool, The Psychosocial Readiness Evaluation and Preparation for Hepatitis C Treatment (PREP-C), which thoroughly assesses the patient's psychosocial readiness to begin treatment and provides a plan to improve readiness. In conjunction with PREP-C, clinics can consider patient followup phone calls, increasing the frequency of appointments, and establishing support groups promoting community and patient engagement.

When discussing adherence and HCV treatment, one disease state that often is subject to controversy is drug abuse. Intravenous drug use (IVDU) is the primary mode of transmission for HCV, and is commonly seen as a factor that reduces adherence resulting in providers limiting access to treatment. By having a well-established relationship with the patient, the healthcare professional can assess the patient as a whole, rather than focusing in on one disease state.

Literature has displayed evidence that patients who inject drugs have demonstrated adherence to prescribed HCVregimens. ${ }^{15}$ In studies containing treatment with IFN adherence and efficacy rates were comparable to those who did not inject drugs. Considering the ease of administration, improved tolerability, and decreased duration of therapy, it can be hypothesized that DAAs may result in even greater adherence rates among IVDU populations in comparison to historic therapies. Within the TLC, drug abuse is not a hard stop to treatment. It merely serves as an indicator for the clinical pharmacist to further assess and determine if treatment is the best option at the current moment. Regardless of HCV treatment, all active drug users encountered are counseled and offered assistance in obtaining help.

In addition to counseling to improve patient adherence and engagement, pharmacy services provide two standardized medication management forms, a personalized medication record (PMR) and a medication action plan (MAP) adapted from the Centers for Medicare and Medicaid Services (CMS). These forms are intended to assist patients in achieving treatment goals. The PMR allows the patient to maintain an update-to-date list of all current medications. Each patient is instructed to carry their PMR with them especially when going to different providers and/or hospitals. Along with the PMR, the MAP is a document mutually agreed upon by the pharmacist and patient that list actions for the patient to take in order to track their progress. At the completion of the first encounter, detailed schedules of all follow-up appointment times are discussed with and given to the patients. It is the sole responsibility of the pharmacist to facilitate medication acquisition through the completion of prior authorizations, schedule follow-up encounters and/or phone calls, and order and assess necessary laboratory work.

\section{Follow-up}

Generally, HCV RNA viral loads are obtained on treatment weeks 4 and 12, and 12 weeks post- treatment. Aside from HCV RNA levels at treatment week 4, a complete blood count $(\mathrm{CBC})$, creatinine level, and hepatic panel are ordered. Patients are notified of their laboratory results as soon as available in EPIC $^{\mathrm{TM}}$. Periodic follow-up phone calls are performed to remind patients of upcoming appointments, provide further adherence counseling, and assess CSDDIs and treatment tolerability. Additional visits may be required if unresolved medication-related problems arise or increased monitoring is necessary.

Clinic discussions with the pharmacist and physician regarding treatment discontinuation occur with every patient who has a detectable HCV RNA level at treatment week 4. Repeat testing on treatment week 6 is usually recommended. As there is no clinic policy or protocol for patients with detectable viral loads, each is discussed on a case-by-case basis. Typically, if the HCV RNA level increases by greater than 10 -fold on repeat testing, the $\mathrm{HCV}$ regimen will be discontinued.

\section{Challenges}

As expected, the development of HCV pharmacy services comes with its share of certain challenges. Many of the $\mathrm{HIV} / \mathrm{HCV}$ co-infected patients are considered inactive, which is defined by the clinic as a patient who has not been seen within the last year. Attempts to reintegrate patients into care can become quite problematic. Contact information listed in the EMR is often out-of-date and cannot be utilized to locate patients. In the circumstance where a patient cannot be reached, the clinic will try to call emergency contacts. Extensive efforts to follow-up with inactive patients are necessary in order to avoid lapses in treatment, disease progression, and further HIV and HCV viral transmission. Once re-integrated, patients must be retained in care, which often presents as an even greater challenge. Empowering patients and giving them ownership of their own health is paramount in having successful retention rates. Rather than the traditional reactive healthcare model, patient-centered models should be utilized placing greater emphasis on provider-patient relationships. Patients must be involved in 
their care plans, and understand the importance of adherence to medications, especially ARVs. One way pharmacists can motivate patients to play a more active role in their health is through education.

The relationship existing between socioeconomic status and health literacy cannot go unacknowledged. Utilizing lay language when providing patient education on disease states in conjunction with the teach-back method is crucial. ${ }^{16,17}$ To ensure effective counseling and assess patient understanding, pharmacists should always ask patients to repeat a point of the counseling session back to them. Commonly in the TLC, lengthier counseling sessions with more frequent follow-up visits are necessary to increase patient disease state knowledge, clinic engagement, and medication adherence. However, the additional time required by the pharmacist to perform extensive counseling and follow-up places a higher demand for resources and pharmacy staff support.

In addition to the increase in staffing demands, finding physician champions has been critical to the development and integration of our services. Despite the involvement of pharmacy services prior to CDTM, clinical pharmacists continue to provide physician education within the institution to discuss the role of the pharmacist in assisting to improve patient outcomes and potentially increase clinic revenue.

As pharmacists broaden their roles in patient-centered care, we anticipate sustainability to be an ongoing challenge.

Recognition by payers is a key component in sustaining clinical services. Although compensation for pharmacy services can be limited, it is the role of the clinical pharmacist to develop a continuing knowledge of CDTM practice billing in order to reduce errors and maintain appropriate levels of reimbursement for provided services. ${ }^{18,19}$ Presently, the clinic is not actively billing for pharmacist ambulatory care services. Majority of the pharmacy visits are done collaboratively with the physician during the medical visit. For clinic revenue purposes, it is not preferred for a pharmacist to see and bill patients on the same day as the physician largely due to CMS paying the lesser of the two bills rather than both claims. ${ }^{20}$ Separate clinic days for pharmacy visits are in development with the addition of pharmacist incident-to billing.

\section{Future Directions}

Currently, the pharmacists in the TLC are conducting a singlecentered, prospective study to evaluate the newly established HCV pharmacy services. In 2015, the clinic treated 22 patients prior to the establishment of CDTM in which 19 patients achieved SVR12 (86\%). Treatment response rates from the study population will be compared to clinic rates in 2015 . Secondary outcomes that will be evaluated include avoidable drug-drug interactions, adherence, care cost/revenue, reported ADRs, and SVR rates according to genotype and stage of fibrosis/cirrhosis. Through the implementation of HCV pharmacy services, we propose that the addition of a clinical pharmacist to an interdisciplinary team treating HIV/HCV coinfected patients will lead to improved SVR rates, decreased medication-related ADRs, and increased adherence, number of drug-drug interactions avoided, and clinic revenue.

As expansion of services to primary care clinics and increased clinic recruitment from hospitalized patients occurs, it is anticipated that there may be some hesitation from providers in referring patients. Education and advertisement of pharmacy clinic services will be developed to potentially curb any uncertainty. In-services to both inpatient and outpatient teams and presentations at medical grand rounds are planned along with interprofessional continuing education lectures. Although there will be a large focus on institutional education, it is the hopes of the clinic to extend its educational efforts to the community.

Many opportunities exist for community outreach in the East New York area given the patient population. As such, the TLC pharmacists plan to provide community educational sessions to aid in reducing the risk of viral transmission and increase screening rates. Of course, this cannot be a one-time session. The medical presence in the community must be sustained in order to build patient-provider relationships and maintain an ongoing trend of education and trust. As the TLC evolves, we hope to leave our mark in the East New York community by serving as public health advocates for this silent epidemic.

\section{Conclusion}

Implementation of HCV pharmacy services for those coinfected with HIV requires a patient- centered strategy in order to address medication-related problems among this unique patient population. Integrating pharmacists into interdisciplinary teams through the establishment of CDTM provides an extra source of support for patients potentially increasing the quality of care received. It is the clinic's belief that the addition of HCV pharmacy services will allow for improved SVR and adherence rates. As the burden of HCVrelated morbidity and mortality continues to increase, clinical pharmacists can rise to the challenge of fighting the silent epidemic by becoming integral members of the medical teams and providing comprehensive medication management services through the development of CPAs.

We declare no conflicts of interest or financial interests that the authors or members of their immediate families have in any product or service discussed in the manuscript, including grants (pending or received), employment, gifts, stock holdings or options, honoraria, consultancies, expert testimony, patents and royalties. 


\section{References}

1. Tsoulfas $G$, Goulis I, Giakoustidis D, et al. Hepatitis C and liver transplantation. Hippokratia. 2009;13(4):211-215.

2. Rein $D B$, Wittenborn JS, Weinbaum CM, Sabin $M$, Smith BD, Lesesne SB. Forecasting the morbidity and mortality associated with prevalent cases of precirrhotic chronic hepatitis $\mathrm{C}$ in the United States. Dig Liver Dis. 2011;43:66-72.

3. Viral Hepatitis. Centers for Disease Control and Prevention.

https://www.cdc.gov/hepatitis/populations/hiv.htm. Published September 26, 2017. Accessed December 12, 2017.

4. Hernandez MD, Sherman KE. HIV/HCV Coinfection Natural History and Disease Progression, A Review of The Most Recent Literature. Current Opinion in HIV and AIDS. 2011;6(6):478-482.

5. Ara AK, Paul JP. New Direct-Acting Antiviral Therapies for Treatment of Chronic Hepatitis C Virus Infection. Gastroenterology \& Hepatology. 2015;11(7):458-466.

6. Woolston SL, Kim N. Lesson 3. Cost and Access to Direct-Acting antiviral Agents. Hepatitis C Online. https://www.hepatitisc.uw.edu/go/evaluationtreatment/cost-access-medications/core-concept/all. Published May 22, 2017. Accessed December 12, 2017.

7. Najafzadeh M, Andersson K, Shrank WH, et al. Costeffectiveness of novel regimens for the treatment of hepatitis C virus. Ann Intern Med. 2015;162:407-19.

8. Langness JA, Nguyen M, Wieland A, Everson GT, Kiser JJ. Optimizing hepatitis $C$ virus treatment through pharmacist interventions: Identification and management of drug-drug interactions. World Journal of Gastroenterology. 2017;23(9):1618-1626.

9. Johnson, et al. Drug-Related Morbidity and Mortality A Cost-of-Illness Model Arch Intern Med. 1995;155(18):1949-1956.

10. Smith JP, Dong MH, Kaunitz JD. Evaluation of a pharmacist-managed hepatitis $\mathrm{C}$ care clinic. Am J Health Syst Pharm. 2007;64(6):632-36.

11. Yang $S$, Britt RB, Hashem MG, et al. Outcomes of Pharmacy-Led Hepatitis C Direct-Acting Antiviral Utilization Management at a Veterans Affairs M. J Manag Care Spec Pharm. 2017;23(3):364-69.

12. N.Y. Education Law $\S \S 6801$ \& 6801-a \& N.Y. Social Services Law §367-A(9)(h).

13. NYC Department of Health and Mental Hygiene. Brooklyn Community Needs Assessment, October 2014.

http://www.health.ny.gov/health_care/medicaid/red esign/dsrip/pps_applications/docs/maimonides_medi cal_center/3.8_maimonides_cna.pdf.
14. NYC HCV Plan. http://natap.org/2016/HCV/012516_01.htm. Accessed December 12, 2017.

15. Lo Re V. Addressing Anticipated Adherence Problems Prior to Treatment. August $10^{\text {th }}, 2017$.

16. "Rodriguez de Bittner M, Sias JJ. Developing Cultural Competency for Self-Care. In: Krinsky DL, ed. Handbook of Nonprescription Drugs."

17. Wawrzyniak AJ, Ownby RL, McCoy K, et al. Health Literacy: Impact on the Health of HIV-Infected Individuals. Current HIV/AIDS reports. 2013;10(4):295-304.

18. Moczygemba LR, Goode J-VR, Gatewood SBS, et al. Integration of collaborative medication therapy management in a safety net patient-centered medical home. Journal of the American Pharmacists Association. 2011;51(2):167-172.

19. McBane SE, Dopp AL, Abe A, et al. Collaborative drug therapy management and comprehensive medication management-2015. Pharmacotherapy. 2015 Apr;35(4):e39-50.

20. Kliethermes MA, Parrott AM, Sachdev G, et al. Pharmacist Billing for Ambulatory Pharmacy Patient Care Services in a Physician- Based Clinic and Other Non-Hospital-Based Environments. https://www.ashp.org/-/media/assets/ambulatorycare-practitioner/docs/sacp-pharmacist-billing-forambulatory-pharmacy-patient-care-services.pdf. Published 2014. Accessed December 12, 2017. 